\title{
Vermittlungsgutscheine als neues Instrument der Arbeitsmarktpolitik: eine erste Analyse
}

\author{
Michael Beckmann \\ Albert-Ludwigs-Universität, Freiburg \\ Julia Deimel \\ Albert-Ludwigs-Universität, Freiburg \\ Bernd Schauenberg \\ Albert-Ludwigs-Universität, Freiburg
}

\section{Einleitung}

Mit Wirkung vom 27. März 2002 hat die Bundesregierung als ein Element eines Programms zur Bekämpfung der Arbeitslosigkeit die so genannten Vermittlungsgutscheine eingeführt. Ziel des Einsatzes dieser Gutscheine, die vom Arbeitsamt an Arbeitslose ausgegeben werden, ist die stärkere Einbindung privater Arbeitsvermittler in die Vermittlung von Arbeitslosen. Die Einführung der Vermittlungsgutscheine muss wohl unter dem Aspekt gesehen werden, dass die Bundesregierung nach der Bemängelung der geschönten Vermittlungsstatistiken der Arbeitsämter durch den Bundesrechnungshof mit einer Sofortmaßnahme schnell agieren wollte. Aber auch die so genannte „HartzKommission" schlägt eine Intensivierung der privaten Vermittlung durch die Weiterentwicklung der Vermittlungsgutscheine vor. Eine erste Bilanz des neuen Arbeitsmarktinstruments zeigt eine recht verhaltene Resonanz. In den ersten sechs Monaten seit Einführung der Vermittlungsgutscheine wurden an 133.195 Arbeitslose von insgesamt 2,5 Millionen Berechtigten Gutscheine ausgegeben. Nur 7.140 Arbeitslose wurden über einen Vermittlungsgutschein in ein Beschäftigungsverhältnis vermittelt. ${ }^{1}$ Angesichts dieser Lage erscheint es

1. Die Daten basieren auf telefonischen Auskünften der Bundesanstalt für Arbeit im Oktober 2002.

(C) Verein für Socialpolitik und Blackwell Publishing Ltd. 2004, 9600 Garsington Road, Oxford OX4 2DQ, UK und 350 Main Street, Malden, MA 02148, USA. 


\section{Michael Beckmann, Julia Deimel und Bernd Schauenberg}

sinnvoll, die Frage aufzuwerfen, was das von der Bundesregierung eingeführte Instrument am Arbeitsmarkt auf längere Sicht leisten kann.

Die weitere Vorgehensweise ist wie folgt. Teil 2 erläutert grob die von der Bundesregierung vorgenommenen Änderungen des arbeitsmarktpolitischen Instrumentariums. Teil 3 skizziert die möglichen ökonomischen Funktionen von Arbeitsvermittlern auf unvollkommenen Arbeitsmärkten mit wechselseitig asymmetrischen Informationsverteilungen. Beide Teile haben vorbereitenden Charakter und werden in Teil 4 verbunden. Es wird erstens geprüft, inwieweit ein bisher schon aktiver Arbeitsvermittler Vermittlungsgutscheine im Rahmen seines bisherigen Tätigkeitsfeldes akzeptiert. Zweitens wird diskutiert, inwieweit ein etablierter Arbeitsvermittler seine Geschäftstätigkeit auf den Markt für Vermittlungsscheine ausweitet. Drittens wird dann gefragt, ob aufgrund der Vermittlungsgutscheine Neugründungen auf dem Markt für Arbeitsvermittler zu erwarten sind. Abschließend werden wir in Teil 5 die Frage prüfen, ob die von der Bundesregierung eingeführten Voraussetzungen bezüglich der Merkmale, die ein Arbeitsloser haben muss, um Anspruch auf einen Vermittlungsgutschein zu haben, die Vermittlungschancen positiv beeinflussen oder nicht.

\section{Die neuen gesetzlichen Regelungen}

Die Grundidee der neuen Regelungen besteht darin, dass ein Arbeitsloser einen privaten Arbeitsvermittler einschalten kann und das Arbeitsamt dazu einen Vermittlungsgutschein ausstellt. Anspruchberechtigt sind Arbeitsuchende, die einen Anspruch auf Arbeitslosengeld oder Arbeitslosenhilfe haben und seit mindestens drei Monaten arbeitslos sind. Der Wert des Gutscheins hängt von der Dauer der Arbeitslosigkeit ab. Bei einer Arbeitslosigkeit von bis zu 6 Monaten ist der Wert auf $1.500 €$, bei 6 bis zu 9 Monaten auf $2.000 €$ und bei mehr als 9 Monaten auf $2.500 €$ begrenzt. Die Gutscheine sind nur drei Monate gültig.

Es gibt zwei Voraussetzungen für die Einlösung der Vermittlungsgutscheine: (1) Der private Arbeitsvermittler muss innerhalb von drei Monaten nachweisen, dass durch seine Tätigkeit ein neues sozialversicherungspflichtiges Beschäftigungsverhältnis zustande gekommen ist. (2) Das Beschäftigungsverhältnis muss eine Mindestdauer von drei Monaten und eine wöchentliche Arbeitszeit von mindestens 15 Stunden aufweisen. Die Auszahlung der Gutscheine erfolgt dann in zwei Raten: (1) Mit dem Beginn des Beschäftigungsverhältnisses werden $1.000 €$ ausbezahlt. (2) Der Restbetrag wird dann ausbezahlt, wenn das Beschäftigungsverhältnis mindestens sechs Monate bestanden hat. Erreicht das Beschäftigungsverhältnis nur eine Dauer von drei bis unter sechs Monaten wird nichts zusätzlich bezahlt. Der Anspruch auf einen Vermittlungsgutschein besteht längstens bis zum 31.12.2004. Bis dahin soll wohl über eine Verlängerung entschieden werden. Wesentlich aber ist, dass aus dieser Regelung zunächst einmal ein Kalkulationszeitraum von 33 Monaten (April 2002 bis Dezember 2004) entsteht. 


\section{Vermittlungsgutscheine: eine erste Analyse}

Neben diesen Regelungen, die sich alle direkt auf die Einführung der Vermittlungsgutscheine beziehen, hat der Gesetzgeber die Gelegenheit genutzt und noch zusätzlich zwei Details der alten gesetzlichen Regelungen von 1994 geändert: (1) Die Erlaubnispflicht zur Betreibung eines Geschäfts der privaten Arbeits- und Ausbildungsvermittlung wurde aufgehoben. (2) Arbeitsvermittler dürfen nun auch von Arbeitsuchenden eine Vermittlungsgebühr bis zu $2.500 €$ verlangen. Bislang durften sie Prämien nur von den potentiellen Arbeitgebern fordern, was auch weiterhin möglich ist. ${ }^{2}$

\section{Private Arbeitsvermittler als Intermediäre}

\subsection{Existenzbedingungen privater Arbeitsvermittler}

Die wissenschaftliche Diskussion um private Arbeitsvermittler ist nicht besonders intensiv. Im Kern gibt es nur zwei wirklich interessante Ansätze. In einem dieser Ansätze wird die Existenz von privaten Arbeitsvermittlern über suchtheoretische Modelle (Bull/Ornati/Tedeschi, 1987), in dem anderen über Transaktionskosten bei Make-or-Buy-Entscheidungen begründet (Föhr, 1995). Beide Ansätze sind sich aber in einem Punkt einig: Private Arbeitsvermittler werden stets als Intermediäre begriffen.

Die Frage nach der Existenz bzw. der Überlebensfähigkeit von privaten Arbeitsvermittlern ist nicht einfach $\mathrm{zu}$ beantworten. Dies ergibt sich vor allem aus der Tatsache, dass die beiden Marktseiten, die für die Existenz der Intermediäre wesentlich sind, selbst tätig werden können. Arbeitnehmer können sich u. a. auf Stellenanzeigen hin bewerben, direkt bei möglichen Arbeitgebern anfragen, soziale Kontakte nutzen oder aber einen Vermittler einschalten. Arbeitgeber können, wenn sie eine freie Stelle zu besetzen haben, in vielen Fällen selbst rekrutieren. Das kann durch interne Suche oder durch die eigene Suche auf dem externen Arbeitsmarkt erfolgen. Erst dann, wenn beide Wege aus welchen Gründen auch immer nicht erfolgreich waren oder nicht beschritten werden sollen, kann ein Arbeitgeber überlegen, einen privaten Arbeitsvermittler einzuschalten. Ob man aus dieser Folge von Schritten schon darauf schließen kann, dass es der private Arbeitsvermittler a priori mit einem vergleichsweise schwierigen Suchproblem zu tun hat, weil zuvor schon zwei Schritte ohne Erfolg geblieben sind, ist denkbar, aber noch nicht abschließend geklärt (Deimel, 1999).

\subsection{Funktionen und Effekte privater Arbeitsvermittler}

Matchingprozesse auf dem Arbeitsmarkt können durch private Arbeitsvermittler in mehrfacher Weise verbessert werden. Ein erster positiver Effekt

2. Vgl. Sozialgesetzbuch (SGB) Drittes Buch (III) - Arbeitsförderung - $§ 241 \mathrm{~g}$ Vermittlungsgutschein, Stand: 27.3.2002. 


\section{Michael Beckmann, Julia Deimel und Bernd Schauenberg}

der privaten Arbeitsvermittlung liegt auf der Hand: Bei ihr könnte man davon ausgehen, dass sie bessere Testergebnisse als eine interne Personalabteilung für Arbeitnehmer mit bestimmten Qualifikationen erzeugt. Dann läge ein klassischer Spezialisierungseffekt vor, der auf eine überlegene Testtechnologie zurückzuführen wäre. Wesentlich ist in diesem Zusammenhang, dass ein privater Mittler auf jeden Fall zu einem früheren Zeitpunkt in seine Fähigkeiten zum Testen von Arbeitnehmern mit bestimmten Eigenschaften investiert haben muss.

Ein zweiter positiver Effekt ergibt sich bei unvorhergesehenem Auftreten einer Vakanz (Bull/Ornati/Tedeschi, 1987). Dadurch, dass die Vermittler ständig testen und mit den getesteten Kandidaten am Markt handeln, stehen die bereits getesteten Arbeitnehmer den Unternehmen bei Bedarf zur Verfügung. Da bei einer Vielzahl von Unternehmen stets eine Vakanz entsteht, geht keine Information über eine getestete Person wieder verloren. Hier verringert der private Vermittler Ausfallschäden, falls der Arbeitgeber zu spät sucht, und versunkene Testkosten, falls der Arbeitgeber zu früh sucht und nicht einstellt. Die Vermittler tragen also dazu bei, die Test- und Ausfallkosten sowie die Kosten für das Horten von Arbeitskräften zu reduzieren. Die Vorteile des privaten Vermittlers gelten vor allem dann, wenn man beispielsweise auf Branchenebene Vakanzen besser als auf Unternehmensebene vorhersehen kann.

Ein dritter positiver Effekt kann sich ergeben, wenn ein informierter privater Arbeitsvermittler dazu beiträgt, Informationsunterschiede zwischen Arbeitgeber und Arbeitnehmer abzubauen. Solche Informationsunterschiede können sich auf verschiedene Faktoren beziehen, u. a. auf Nicht-LohnMerkmale eines Arbeitsvertrags, auf betriebliche Lohnstrukturen, aber auch auf schwer prüfbare Determinanten der Qualifikation des Arbeitnehmers. Gerade unter solchen Informationsbedingungen sollten die Vorteile von Intermediären zum Tragen kommen.

Einen letzten positiven Effekt kann man als Folge des dritten Effektes betrachten. Ein privater Arbeitsvermittler wird dauerhaft mit beiden Marktseiten Kontakt halten müssen. Unter diesen Bedingungen wird er mit hoher Wahrscheinlichkeit schneller als die anderen Akteure auf neue Trends stoßen. Wenn z. B. die Personalabteilung eines Unternehmens zum ersten Mal Arbeitnehmer mit einer neuen Qualifikation sucht, dann wird sie nicht erkennen können, ob andere Unternehmen gleichzeitig vor ähnlichen Problemen stehen. Ein Mittler aber sollte dazu in der Lage sein, solche Zusammenhänge zu erkennen und zu seinem Vorteil zu nutzen.

\section{Private Arbeitsvermittler und Vermittlungsgutscheine}

\subsection{Ausweitung der Geschäftstätigkeit bei aktiven Vermittlern}

Wir wollen zunächst der Frage nachgehen, ob ein schon aktiver Intermediär auch auf dem Markt für Vermittlungsgutscheine aktiv werden wird. Dies ist erst 


\section{Vermittlungsgutscheine: eine erste Analyse}

dann möglich, wenn die Situation vor der Einführung der Vermittlungsgutscheine präzisiert worden ist. Zwei Voraussetzungen wird man bedenken müssen. Erfolgreiche Vermittler werden sich erstens darauf spezialisiert haben, die Qualitäten bestimmter Arbeitnehmer zu testen. ${ }^{3}$ Für den Erwerb dieser Fähigkeit werden Fixkosten in nicht unbeachtlicher Höhe angefallen sein. Sie werden sich zweitens auch auf bestimmte Kunden hin spezialisiert haben. Für die Fähigkeit, deren Nachfragebedingungen sicher beurteilen zu können, werden sie beziehungsspezifische Investitionen in wiederum nicht unbeachtlicher Höhe aufgewendet haben müssen. Auch diese werden zu heute versunkenen Fixkosten geführt haben.

Wegen der starken Fixkosteneffekte wird ein erfolgreicher Vermittler schon vor der Einführung der Vermittlungsgutscheine einen starken Anreiz gehabt haben, die für ihn möglichen Skalenerträge zu nutzen. Im Zentrum seiner Überlegungen wird dabei die Frage gestanden haben, wie der Pool an Arbeitnehmern mit den für seine Spezialisierungsstrategie relevanten Qualifikationen möglichst stark auszuweiten ist. Eine Grenze dafür wird sich dann ergeben haben, wenn die Chance, weitere geeignete Kandidaten zu finden, so klein geworden ist, dass der Vermittler nicht mehr bereit war, die vergleichsweise sicheren zusätzlichen Kosten für das Ausweiten des Pools und das zusätzliche Testen zu tragen. Dann aber wird man mit großer Wahrscheinlichkeit davon ausgehen müssen, dass sich der Pool an Kandidaten eines Vermittlers allein durch die Einführung der Vermittlungsgutscheine nicht vergrößern wird. Denkbar sind lediglich zwei wenig wahrscheinliche Ausnahmefälle. Für den Fall, dass der Vermittler zusätzliche Kandidaten findet, dürften diese seine Tests kaum bestehen. Denkbar wäre es noch, dass der Vermittler seine Anforderungen an die Kandidaten senkt. Das ist jedoch unplausibel, denn dann wird er seinen bisherigen Kunden schlechtere Kandidaten als bislang vermitteln und einen Reputationsverlust erleiden.

Schließlich hat auch der Wert der Gutscheine Konsequenzen für das Verhalten der Akteure. Die bisherige Honorargestaltung sah vor, dass der private Personalvermittler vom neuen Arbeitgeber meist 2 bis 2,5 Bruttomonatsgehälter des vermittelten Arbeitnehmers als Vermittlungsprovision erhielt. Entscheidend für eine ökonomische Analyse ist das Verhältnis zwischen dem Wert des Gutscheins W und dem Marktpreis für die Vermittlung P. Gilt W $>$ P, dann sind die Konsequenzen für die Vermittler unklar. Zunächst einmal wird es einen starken Anreiz für die Vermittler geben, sich auf das Geschäft mit den Vermittlungsgutscheinen zu konzentrieren. Naheliegend ist dann der oben angedeutete Substitutionseffekt: Vermittlungsgeschäfte, die ansonsten über den Markt abgewickelt worden wären, laufen jetzt über die Vermittlungsgutscheine. Vermittler und ihre Kunden realisieren Mitnahmeeffekte. Nicht auszuschließen ist zudem, dass die Vermittler einen Anreiz haben, schlechte Kandidaten mit Gutschein besseren Kandidaten ohne Gutschein vorzuziehen.

3. Vgl. dazu die Befunde bei Johst (2000) für Deutschland und Vosberg (2001) für die USA.

(C) Verein für Socialpolitik und Blackwell Publishing Ltd. 2004 


\section{Michael Beckmann, Julia Deimel und Bernd Schauenberg}

Gilt hingegen $\mathrm{W}<\mathrm{P}$, dann werden sich ausgelastete Vermittler nicht an dem Programm beteiligen oder ihr Verhalten am Markt ändern. Genau dieser Effekt wird in den Monaten seit Einführung der Gutscheine beobachtet. Die Vermittler beklagen die Deckelung der Gutscheine unterhalb der marktüblichen Prämie (o. V., 2002a). In der Praxis wird nun eine "Cofinanzierung" mit den Gutscheinen betrieben. Die Arbeitgeber zahlen grundsätzlich den Marktpreis für die Vermittlung. Besitzt der vermittelte Kandidat einen Vermittlungsgutschein wird die Provision um den Nennbetrag des Gutscheines gekürzt. Die Arbeitgeber zahlen P - W an die privaten Vermittler. Diese erhalten ihre Provision so teils vom Arbeitsamt und teils vom Kunden. Die Einlösung der Vermittlungsgutscheine beim Arbeitsamt ist dabei für die Vermittler nicht unproblematisch. So ist im Vergleich zu den bisherigen Zahlungsmodalitäten für Vermittlungsdienstleistungen der administrative Aufwand deutlich gestiegen. Das Interesse der privaten Vermittler an der Gutscheinregelung wird dadurch noch geringer. Da die Arbeitgeber für die Vermittlung von Arbeitslosen nun aber bezahlen müssen, wird auch auf dieser Seite die Attraktivität der Vermittlungsgutscheine geschmälert. Die Hartz-Kommission macht für die Weiterentwicklung der Vermittlungsgutscheine keine konkreten Aussagen, sondern schlägt lediglich eine Anpassung der Förderhöhe an die Markterfordernisse vor (Hartz-Kommission, 2002, S. 62). Eine flexiblere Gestaltung der Gutscheinwerte ist nach den Überlegungen auf jeden Fall geboten.

\subsection{Anreize für Investitionen in neue Testtechnologien und Marktneueintritte}

Wegen der finanziellen Anreize aus dem Programm der Bundesregierung könnte ein schon aktiver Mittler überlegen, ob er neue Testfähigkeiten entwickeln soll, um sein Geschäftsfeld auszuweiten. Ein solcher Intermediär steht also vor einem Investitionsproblem. Im einfachsten Fall führt die Entwicklung der neuen Testfähigkeiten in einer ersten Periode zu Anschaffungsauszahlungen in gegebener Höhe und zu positiven Einzahlungsüberschüssen aus neuen Vermittlungsgeschäften in den nachfolgenden Perioden. Wenn der Kapitalwert dieser Investition positiv ist, wird der Vermittler die Investition tätigen. Ob dies der Fall ist, hängt wesentlich von der erwarteten Nutzungsdauer der neuen Testfähigkeiten ab. Die Tatsache, dass die Bundesregierung das neue Programm längstens auf das Ende von 2004 begrenzt hat, ist für dieses Vorteilhaftigkeitskalkül sicherlich hinderlich.

$\mathrm{Zu}$ prüfen ist weiterhin, ob es infolge des Programms der Bundesregierung auf dem Markt für Arbeitsvermittler zu Neueintritten kommen wird. Das aber erscheint sehr unwahrscheinlich, denn in diesem Fall gelten alle Überlegungen, die oben für die Erweiterungsinvestitionen etablierter Anbieter angestellt wurden. Auch hier kann es bestenfalls marginale Effekte bei denjenigen Akteuren geben, die sich schon fast für einen Markteintritt entschieden haben. Ein möglicher positiver Effekt ist jedoch bei Anbietern anderer 


\section{Vermittlungsgutscheine: eine erste Analyse}

Personaldienstleistungen zu sehen. So könnten Unternehmen, die bislang Weiterbildungsleistungen angeboten haben, über die Vermittlungsgutscheine in die Vermittlung der von ihnen geschulten Personen einsteigen. Die Vermittlung würde als Nebengeschäft ausgeübt, das die Unternehmen zusätzlich zu der eigentlichen Tätigkeit anbieten. Die Personaldienstleister könnten zusätzlich von der anderen Gesetzesneuerung profitieren. Durch die Abschaffung der Erlaubnispflicht für private Arbeitsvermittler wird der Markteintritt neuer Anbieter erleichtert. Allerdings kann dies auch zu vermehrten Neueintritten unseriöser Anbieter führen.

\subsection{Test auf allgemeine Qualifikationen}

Bislang wurde stets unterstellt, dass ein privater Arbeitsvermittler auf berufsoder branchenspezifisches Humankapital testet. Denkbar wäre es jetzt, dass Mittler den Versuch unternehmen, auf allgemeinere Qualifikationen zu testen. Dann wäre wohl mit geringeren Entwicklungskosten für Tests zu rechnen. Ziel könnte es z. B. sein, bei Langzeitarbeitslosen zu testen, ob sie im Unternehmen überhaupt einsetzbar sind oder ob bei einzelnen Kandidaten negative Eigenschaften vorliegen, die Langzeitarbeitslosen als Stigma anhaften, wie z. B. Unzuverlässigkeit oder geringes Engagement. Im Prinzip wäre eine derartige Differenzierung durch entsprechende Tests möglich. Unklar aber ist, ob es solche Tests überhaupt gibt und ob sie für eine erfolgreiche Vermittlung überhaupt hinreichend sind. Die Tatsache, dass sie bisher noch kaum beobachtet wurden, spricht jedenfalls entschieden gegen diese Möglichkeit.

\subsection{Manipulationsanreize}

Die Bedingungen, die zur Auszahlung eines Gutscheins führen, sind offenbar nur schwer $\mathrm{zu}$ kontrollieren und laden so $\mathrm{zu}$ Manipulationen ein. Das Arbeitsamt kann kaum beobachten, ob die Vermittlung eventuell durch die Kollusion zwischen Arbeitslosem und Vermittler mit dem Ziel, den ersten Teil der Prämie zu kassieren, erfolgte. Das Arbeitsverhältnis würde dann nach Auszahlung der Prämie sofort wieder aufgelöst. Auch könnten Arbeitgeber und Vermittler gemeinsam Einstellungen fingieren und so die Prämien abschöpfen. Mittler könnten also auf die Idee kommen, die Gutscheine mit Unternehmen oder Arbeitsuchenden zu „teilen“. ${ }^{4}$

Der Umstand, dass parallel zur Einführung der Vermittlungsgutscheine die Erlaubnispflicht privater Arbeitsvermittler wegfällt, macht den Markt unübersichtlicher und verschärft so das Problem. Dies schädigt nicht nur den Ruf der

4. Erste Versuche sind schon bekannt geworden. So wurde Arbeitslosen auf einer Internetseite angeboten, $500 €$ des Gutscheins bei Arbeitsaufnahme behalten zu dürfen. Dies führte dann dazu, dass Arbeitslose von seriösen Vermittlern bei erfolgreicher Vermittlung die Herausgabe eines Teils der Prämie verlangten (o. V., 2002b). 


\section{Michael Beckmann, Julia Deimel und Bernd Schauenberg}

gesamten Branche, sondern trägt auch zur Verunsicherung der Arbeitslosen bei, die sich an die Vermittler wenden. Da die Arbeitsämter aus Gründen der Wettbewerbsgleichheit mit der Ausgabe des Gutscheines keine Angaben über seriöse Vermittler machen dürfen, stehen die Arbeitslosen ohnehin recht orientierungslos da. Insofern besteht auch ein Anreiz unseriöser Personalvermittler, den erleichterten Markteintritt für Mitnahmeeffekte zu realisieren, ohne ein langfristiges Interesse zu haben.

\section{Problemgruppen des Arbeitsmarktes und private Vermittlung}

$\mathrm{Zu}$ einer ökonomischen Einschätzung der künftigen Erfolgspotentiale einer Privatisierung der Arbeitsvermittlung bei der Bekämpfung der Arbeitslosigkeit gehört schließlich auch eine Analyse der Struktur der zu vermittelnden Arbeitsuchenden. Bisher haben sich private Arbeitsvermittler vor allem auf die Vermittlung qualifizierter Arbeitnehmer konzentriert. Gefördert wurde diese Fokussierung maßgeblich durch Provisionsregelungen, die sich allein nach dem Verdienst der Vermittelten richteten. Bevorzugte Kandidaten für eine private Arbeitsvermittlung waren damit vergleichsweise einfach $\mathrm{zu}$ vermittelnde Arbeitskräfte.

Mit der Erteilung von Vermittlungsgutscheinen an Arbeitslose ändert sich das potentielle Klientel privater Arbeitsvermittler auf der Arbeitsangebotsseite nun dramatisch. Geht es nach den Vorstellungen der Reformer, wird die private Arbeitsvermittlung nun in großem Maße mit den so genannten Problemgruppen des Arbeitsmarktes konfrontiert werden. Die so bezeichneten Arbeitnehmergruppen spielen in Deutschland eine erhebliche Rolle in den Arbeitslosenstatistiken und bedurften in der Vergangenheit als „schwer vermittelbare Arbeitskräfte" besonderen Vermittlungsanstrengungen vonseiten der Arbeitsämter. Die entscheidende Frage bei einer stärkeren Privatisierung der Vermittlungsleistungen ist nun also, ob und inwieweit dadurch eine Steigerung der Vermittlungseffizienz für den Personenkreis der Problemgruppen erwartet werden kann. Im weiteren Verlauf sollen zur Verdeutlichung der Argumentation lediglich Arbeitnehmer ohne abgeschlossene Berufsausbildung, ältere Arbeitnehmer sowie Langzeitarbeitslose betrachtet werden.

\subsection{Gering Qualifizierte}

Arbeitnehmer ohne einen Ausbildungsabschluss gehören seit geraumer Zeit zu den schwer vermittelbaren Arbeitskräften. Im Zuge technologischer Innovationen und einer zunehmenden Globalisierung verschwinden Tätigkeitsfelder für gering Qualifizierte immer mehr aus den Unternehmen. Neue Arbeitsplätze mit höheren Anforderungen entstehen, für die unqualifizierte Mitarbeiter nun allerdings kaum noch infrage kommen (Chennells/Van Reenen, 1999; Nickel/Bell, 1995). Die Vermittlung von Arbeitskräften ohne 


\section{Vermittlungsgutscheine: eine erste Analyse}

Berufsabschluss ist also mit einer immer geringeren Nachfrage der Unternehmen konfrontiert. Dies hat sich schon für die Arbeitsämter als großes Problem erwiesen, welches auch durch eine stärkere Privatisierung der Arbeitsvermittlung nicht geringer wird. Fraglich ist außerdem, ob für den privaten Personalvermittler überhaupt ein ausreichender Anreiz besteht, sein Klientel um Arbeitskräfte ohne Berufsabschluss, die ja geringste Einstiegslöhne zu erwarten haben, zu erweitern. Durch die Vermittlungsgutscheine sollte nun eine weitere Einnahmequelle für den Personalvermittler hinzu kommen. Aber ob die Gesamtkompensation die erforderlichen Vermittlungsaufwendungen rechtfertigt, darf weiterhin bezweifelt werden. Erste praktische Erfahrungen sprechen jedenfalls nicht dafür (o. V., 2002c). Hinzu kommt das schon oben angesprochenes Problem, dass gering qualifizierte Arbeitslose im Kontext gestiegener Arbeitsanforderungen die Tests der Personalvermittler vermutlich nicht bestehen.

Aber selbst wenn man von einer gegebenen Arbeitsnachfrage nach gering Qualifizierten und einer Vermittlungsbereitschaft der Privaten ausgehen könnte, dürfte das Interesse der Unternehmen an einer privaten Arbeitsvermittlung eher gering sein. Nach einer Untersuchung von Blaschke (1987) erhalten Arbeitnehmer ohne abgeschlossene Berufsausbildung, sofern sie eine Stelle finden, diese sehr häufig über soziale Kontakte. Dieser Umstand könnte einerseits zwar als Chance für private Arbeitsvermittler angesehen werden, an die Stelle sozialer Kontakte zu treten. Andererseits deutet das Zustandekommen von Arbeitsverhältnissen durch soziale Kontakte aber auch darauf hin, dass das Unternehmen bei der Besetzung unqualifizierter Arbeitsplätze kein besonderes Matching-Problem vermutet. Insofern kann dann auch auf ein intensives Testen verzichtet werden. Die Einschaltung eines externen Personalberaters kann daher entfallen und die Arbeitskräftebeschaffung bei Bedarf durch die Unternehmen selbst vergleichsweise einfach vorgenommen werden.

\section{2 Ältere Arbeitnehmer und Langzeitarbeitslosigkeit}

Ältere Arbeitnehmer ab 50 Jahren haben im Vergleich zu Arbeitslosen anderer Altersgruppen die höchsten Verweildauern in der Arbeitslosigkeit. Sie finden selbst bei entsprechender beruflicher Qualifikation viel schwerer eine neue Beschäftigung als jüngere Arbeitslose und stellen damit auch den Hauptbestandteil der Langzeitarbeitslosen dar, gefolgt von den gering Qualifizierten (Bundesanstalt für Arbeit, 2001, S. 196-198). Als Gründe für die Vorbehalte von Unternehmen gegen eine Einstellung älterer Mitarbeiter können relativ hohe Löhne, ausgeprägte Kündigungsschutzregeln, eine abnehmende Produktivität, z. B. aufgrund von Humankapitalabschreibungen, sowie die geringe erwartete Restverweildauer im Betrieb, die Humankapitalinvestitionen wenig attraktiv erscheinen lässt, genannt werden. Somit kommt sowohl für ältere als auch für gering qualifizierte Arbeitslose neben diesem ersten „Handicap“ mit der Langzeitarbeitslosigkeit oftmals noch ein weiteres Einstellungshemmnis und ein weiteres Kriterium für eine schwere Vermittelbarkeit hinzu. 


\section{Michael Beckmann, Julia Deimel und Bernd Schauenberg}

Aus der Sicht der Unternehmen ist die Einstellung von Langzeitarbeitslosen nicht unproblematisch. Es ist denkbar, dass Langzeitarbeitslosigkeit den Betroffenen als Stigma anhaftet. Arbeitgeber könnten daraus auf eine nur geringe Qualität oder andere negativ belegte Eigenschaften der Stellenbewerber schließen. Wenn die Unternehmen Langzeitarbeitslosigkeit tatsächlich als ein derartiges Signal auffassen, muss davon ausgegangen werden, dass ihre Bereitschaft, Arbeitslose neu einzustellen, bei Personen mit mehreren „Handicaps" weiter abnimmt. Ein privater Arbeitsvermittler könnte vielleicht sogar eher als ein öffentlicher ein vorhandenes Misstrauen der Arbeitgeber gegenüber Langzeitarbeitslosen abbauen, jedoch stünden einer erfolgreichen Vermittlung dann immer noch die Kriterien Alter bzw. mangelnde Qualifikation entgegen.

\section{Zusammenfassung und Ausblick}

Die zusammenfassende Beurteilung einer verstärkten Privatisierung der Arbeitsvermittlung über das System der Vermittlungsgutscheine fällt eher pessimistisch aus. Zwei Gründe sind für dieses Urteil verantwortlich: Erstens muss zumindest infrage gestellt werden, ob private Arbeitsvermittler durch das neue System überhaupt einen ausreichenden Anreiz haben, ihre Vermittlungsleistungen auf die Problemgruppen des Arbeitsmarktes auszuweiten. Die Vermittlungsgutscheine stellen zwar eine zusätzliche Entlohnungskomponente für den privaten Arbeitsvermittler dar. Dennoch bestehen Zweifel daran, dass die zu tätigenden Investitionen und die Vermittlungsaufwendungen für die Problemgruppen in einem angemessenen Verhältnis zu den realisierbaren Zusatzerträgen stehen. Zweitens sind die hier skizzierten Problemgruppen des Arbeitsmarktes nicht deshalb Problemgruppen und schwer vermittelbar, weil die bisherige Vermittlung über die Arbeitsämter besonders ineffizient organisiert war, sondern aufgrund divergierender Qualifikations- und Anforderungsprofile von Arbeitsanbietern bzw. -nachfragern. Es sind also vor allem strukturelle Probleme, die es zu überwinden gilt. Diese werden aber nicht dadurch gelöst, dass man vormals öffentliche Vermittlungsleistungen nun privatisiert. Die privaten Arbeitsvermittler werden nach wie vor im Kundenauftrag tätig. Durch den öffentlichen Auftrag, nun auch Problemgruppen $\mathrm{zu}$ vermitteln, werden sie von ihrem Prinzip, ihren Kunden stets den bestmöglichen Kandidaten zu vermitteln, aufgrund des Reputationsverlustes bei Vermittlung eines ungeeigneten Kandidaten, nicht abweichen. Die HartzKommission nennt nur zwei Vorschläge zum Thema Vermittlungsgutscheine. Zum einen ist es die erwähnte Anpassung der Förderhöhen in Abhängigkeit der Vermittlungshemmnisse an die Markterfordernisse. Zum anderen sollen Mitnahmeeffekte durch die Konzentration auf Schwervermittelbare und ein aussagefähiges Profiling der Arbeitsuchenden verringert werden (HartzKommission, 2002, S. 62). Diese Modifizierungen sind leichte Verbesserungen der in unserer Analyse aufgezeigten Problempunkte. Nach unserer Einschätzung werden jedoch auch diese Korrekturen den Vermittlungsgutscheinen nicht zu 


\section{Vermittlungsgutscheine: eine erste Analyse}

der gewünschten Effektivität verhelfen. Der Versuch einer Stärkung der privaten Vermittlung von Arbeitslosen wird durch die Vermittlungsgutscheine vermutlich nicht gelingen und das Arbeitslosenproblem somit nicht verringert. Unsere Analyse zeigt im Kern ein Handeln nach dem Motto „Therapie ohne Diagnose".

\section{Literaturverzeichnis}

Blaschke, D. (1987), Erfolgswege zum neuen Arbeitsplatz, Mitteilungen aus der Arbeitsmarkt- und Berufsforschung 20, 164-180.

Bull, C., Ornati, O., und Tedeschi, P. (1987), Search, Hiring Strategies, and Labor Market Intermediaries, Journal of Labor Economics 5, 1-17.

Bundesanstalt für Arbeit (Hrsg.) (2001), Arbeitsmarkt 2000: Amtliche Nachrichten der Bundesanstalt für Arbeit, 49. Jg. Sondernummer, Nürnberg, 28. Juni.

Chennells, L. und Van Reenen, J (1999), Has Technology Hurt Less Skilled Workers? An Econometric Survey of the Effects of Technical Change on the Structure of Pay and Jobs, Working Paper No. W99/27, Institute for Fiscal Studies.

Deimel, J. (1999), Ökonomische Analyse der Rekrutierung von Führungskräften, unveröffentlichte Diplomarbeit, Universität Freiburg.

Föhr, S. (1995), Personalberatung als Institution: Make or Buy-Entscheidungen im Personalbereich, Zeitschrift für Personalforschung 9, 135-162.

Hartz-Kommission (2002), Moderne Dienstleistungen am Arbeitsmarkt, Berlin.

Johst, D. (2000), Angebot an Personaldienstleistungen in Deutschland - eine theoretische und empirische Analyse, Jahrbücher für Nationalökonomie und Statistik 220, 165-190.

Nickel, S. und Bell, B. (1995), The Collapse in Demand for the Unskilled and Unemployed Across the OECD, Oxford Review of Economic Policy 11, 40-62.

o. V. (2002a), Gutscheine für Jobvermittlung werden gedeckelt. Höhe der Vergütung für private Vermittler unter Marktniveau, Financial Times Deutschland, 14 März, 12.

o. V. (2002b), Geteiltes Honorar oder die Verlotterung der Sitten, Frankfurter Rundschau, 8 Mai, 9.

o. V. (2002c), Neue Jobvermittlung ein Flop, Süddeutsche Zeitung, 5 April, 37.

Vosberg, D. (2001), Die Entwicklung der Personaldienstleistungsbranche in den USA eine explorative Studie, Jahrbücher für Nationalökonomie und Statistik 221, 202-225.

Abstract: This paper gives a first appraisal of the "Vermittlungsgutscheine" the German government has recently created as a new "instrument of labor market policy. This instrument is aimed at improving intermediation of unemployed people by inciting private recruiters to place individuals who were formerly advised by the state-run placement-agencies. We analyze the function of the placement intermediaries and discuss how they will respond to the new measure. It is argued that, mainly due to the amount and the short term of the tokens, private intermediaries will not participate in this programme significantly. We also look at the labour market and find that the unemployed individuals mostly do not match the skills the employers are looking for. Thus, we do not think that this instrument will have a noticeable effect on the labour market. 
Brought to you by | Universitaetsbibliothek Basel

Authenticated 